\title{
Metrological Approach to Measurements of Emotions Being Expected in Response to Acoustic Impacts
}

\author{
Kseniia Sapozhnikova ${ }^{1,}$, Roald Taymanov ${ }^{1}$, Iuliia Baksheeva $^{2}$, Svetlana Kostromina ${ }^{3}$, Daria Gnedykh $^{3}$, Irina Danilova $^{1}$ \\ ${ }^{1}$ D. I. Mendeleyev Institute for Metrology, 19 Moskovsky pr., St. Petersburg, Russia, 190005 \\ ${ }^{2}$ St. Petersburg State University of Aerospace Instrumentation, 67 Bolshaya Morskaya str., St. Petersburg, Russia, 190000 \\ ${ }^{3}$ St. Petersburg State University, The Department of Psychology, 7/9 Universitetskaya emb., St. Petersburg, 199034
}

\begin{abstract}
The paper deals with the task of measuring emotions that most likely arise as a result of certain acoustic impacts. This task is of interest for musicology, philology, medicine, robotics, etc. A measurement model is presented that reflects a "mechanism" of forming the emotions. Analysis of evolution of animal's bioacoustic signals, calculations, and experiments have made it possible to justify the model. The subjectivity of well-known emotion scales is shown. For the model, a new scale is proposed and grounded that can provide the metrological traceability of measurement results. The model enables explaining changes in emotional perception of acoustic impacts. A method is proposed to diagnose infant's emotional development based on sounds of cry and other vocalizations.
\end{abstract}

\section{Introduction}

Emotion is a sensory reaction that arises in a subject in response to a new impact. Emotion (proto-emotion) ability has emerged together with life origination and can be considered as its identifier.

According to $[1,2]$, emotions motivate, organize and direct perception, thinking and actions, contributing to preservation and development of population. In the course of human evolution and development of society, the number of emotions has been increased.

In addition to the most ancient emotions, inherent in humans and animals, emotions have arisen that are characteristic only for people, and then emotions have originated distinguishing some socio-cultural groups of the population from others.

Naturally, any emotion is preceded by a certain neurophysiological reaction that can be the simplest or a complex one.

The emotion, arising most likely in humans and animals under an acoustic impact, i.e. the expected one, is one of the multi-dimensional (multiparametric) quantities, measurements of which (in a strictly metrological interpretation) became of current interest in the 21 st century.

Measurements of expected emotions can help a performer of a musical composition in the process of rehearsals to take into account the national-cultural and social specifics of probable listeners in a better way and, thereby, achieve a greater success.

For a philologist, measurements of emotions in speech sounds will allow students studying a foreign language to realize the peculiarities of its tonal coloring.

In the future, development of efficient measuring systems performing the automatic simultaneous interpretation of speech from one language to another, will be impossible without embedding instruments measuring emotions. Taking into account the results of such measurements, it will be necessary to select synonyms required and to clarify a phrase structure.

Emotion measurements will be needed while developing robots capable to active communication with human.

The solution of the listed tasks and a number of others related to the results of measuring the emotions that arise (with the greatest probability) in response to acoustic impacts, has became possible due to the development of an appropriate measurement model.

In the process of research, the emotion was defined by the authors of this paper as a neurophysiological reaction (below, this reaction will be called emotiogenic one) due to some impact, which causes the transition of a person to a state that allows us to identify the emotion character by the sensation or behavior of a person.

Initially, this model contained one step and was justified by the results of a theoretical analysis of the evolution of biolinguistic signals and differences between the major and minor keys. Later on, the model was supplemented by the second and third steps, and its justification was strengthened by experiments performed with the participation of neurophysiologists, psychologists, and musicians.

These experiments included:

- analysis of the correspondence between listener's encephalograms obtained under the influence of certain sounds and those reactions that the model predicted.

- study of the correspondence between bio-linguistic signals of animals and the interpretation, which follows from the impact results of the according to the model;

* Corresponding author: author@e-mail.org

(C) The Authors, published by EDP Sciences. This is an open access article distributed under the terms of the Creative Commons Attribution License 4.0 (http://creativecommons.org/licenses/by/4.0/). 
- experiment- calculated analysis of excerpts of ethnic and bell music, etc.

The developed measurement model can be considered as a model of the "mechanism" of forming the emotions. That is why it allows us to explain some effects in the field of neurophysiology, psychology, and musicology.

\section{Emotion scales}

The measurement model consists of 3 steps. Its 1st step demonstrates the "mechanism" of the basic emotion formation. It is shown in Fig. 1 and contains a preselector of sound range oscillations (the preselector reflects an auricle with a membrane), nonlinear converter, delay unit (based on an operative memory), frequency selector of the sounding energy maximum zone, selector of oscillations with frequencies in the zone of the brain biorhythms, as well as units that provide the identification of basic emotions.

Complex emotions named here as emotional images are formed in subsequent steps of the model. In the 2nd step, the basic emotional images (BEI) that have a general human character are generated, and in the 3rd step the formation of culture dependent emotional images (CEI), takes place (Fig. 2). They are associated with the cognitive human activity, upbringing, education, national history, as well as belonging to a certain socio-cultural group.

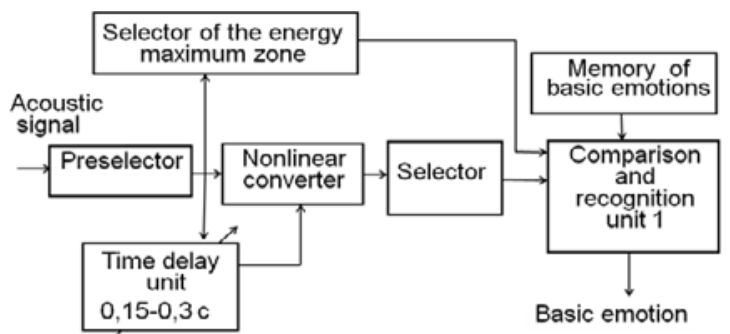

Fig. 1. The 1st step of the measurement model [3].

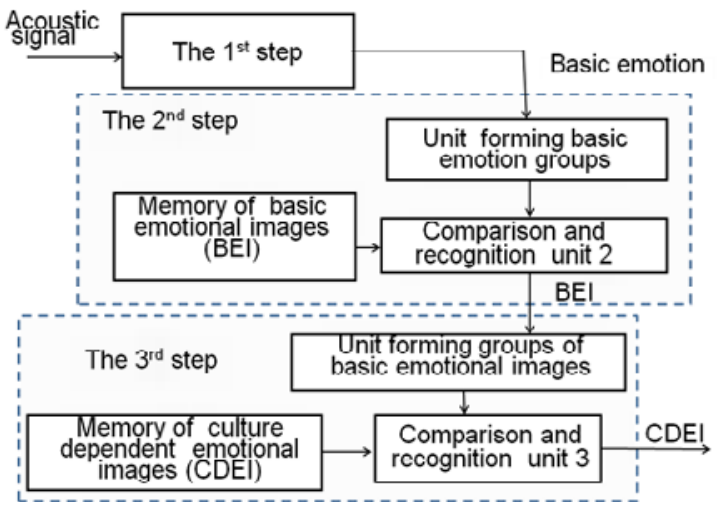

Fig. 2. Three-steps measurement model [3].

Calculation-experimental studies were performed with the participation of neurophysiologists, psychologists, mathematicians, metrologists, an art- therapist, and a composer. These investigations have confirmed that the proposed model corresponds to the processes that occur when emotions are generated in response to a certain acoustic stimulus [3-10].

It follows from the analysis of the model given above that the earliest (the most ancient) emotions that are usually called basic ones, are associated with the frequencies of the brain biorhythms, their difference being identified by the frequencies of delta-, theta-, or alpha-brain biorhythms. The enrichment of emotion spectrum is connected, firstly, with combination of the corresponding biorhythm frequency and a sound carrier frequency; secondly, with simultaneous excitation of 2-3 biorhythms, thirdly, with the sequence of several biorhythm groups.

The scale of basic emotions proposed by Izard [1] includes 10 gradations: "anger", "contempt", "disgust", “distress", "fear", "guilt", "interest”, "joy”, "shame”, and "surprise". Ekman in [11] singles out 6 gradations: "fear", "anger", "disgust", "sadness", and "happiness". In [12] 8 basic emotions are proposed: "anger" - "fear", "approval" - "disgust", "happiness" - "sadness", "expectation" - "surprise". Kulikov in [13] notes the necessity to include "excitement" ("gambling") and "vigour". In the Ilyin's opinion, "shame", guilt", and "interest" should be excluded from the list [14].

A unified idea of the basic emotion scale has not been formed until present. Subjectivity and inconsistency of the scales considered above, in our view, is caused by the fact that their authors did not rely upon the concept of bioacoustic signals of living creatures in the course of evolution.

The measurement model can be used as a model of emotion formation "mechanism" as well. This statement is the result of the analysis of bioacoustic signal evolution and evolution of basic emotions colouring these bioacoustic signals. The correspondence between these signals and basic emotions was obtained in experiments [15]. This basis enables connecting "threat emotion", which was not mentioned above, with the neurophysiological reaction within the delta-rhythm zone.

Since, as it follows from the model, an expected emotion potentially contained in sounding is associated with both a modulating infrasound component frequency and carrier frequency, it is possible to divide this gradation into two parts "fear" and "anger" (fearsome emotion). At the same time it should be noted that:

- high carrier frequency (higher than, approximately, $10 \mathrm{kHz}$ ) is characteristic for a sounding aimed at warning somebody against danger in the maximum large space (this is "fear");

- low carrier frequency (lower than, approximately, 1 $\mathrm{kHz}$ ) indentifies the emotion addressed to somebody being at a close vicinity (this is "anger").

Similarly, it is logical to define the neurophysiological reaction with the theta-rhythm frequency as reflex emotion "alertness" singling out "surprise" and "expectation" in accordance with a carrier frequency.

Finally, the neurophysiological reaction with the alpha-rhythm frequency can be identified as reflex 
emotion "exitation" with its possible manifestations (stimulated by a carrier frequency) such as "happiness" and singling out "passion" ("gambling").

The proposed structure of basic emotion scale provides the metrological traceability (of course, within the uncertainty of biorhythm boundaries) while measuring expected emotions. It is characteristic that the number of emotions here is limited by the number of the brain biorhythms that participate in their formation, but it approximately corresponds to the number of basic emotions, considered by some psychologists using other motives.

In accordance with the model, the total number of emotions that are common for human and developed animals (mammals) is significantly higher than 6 , since it is determined by the number of possible combinations of a few (usually, up to 3) basic emotions. It is interesting that many years ago Plutchik came to the same idea [12], but his choice of basic emotions has no justification.

It should be noticed that the basic emotion scales are not the only scale type applied in investigations of subjective perception of such acoustic impacts as music.

One-dimensional (discrete) scales are well-known that include up to 45 emotions [16, 17].

However, the choice of emotions is subjective and does not take into account the influence of a specific culture on emotional perception. In the view of the present paper authors, the development of scales with a great number of gradations is reasonable only for complicated emotions, emerging as a result of consecutive origination of basic emotion groups. Taking into account that time of emotion origination is very short, the complicated emotion formation can be considered as the process of origination of a feeling, i.e., integral estimating reaction caused by emotional perception of acoustic impacts.

Other one-dimensional scales are given in [18, 19]. Multi-dimensional scales are widely used as well [19, $20]$.

Drawbacks of all these scales are as follows:

- Non-equivalent reactions are integrated, i.e., emotions of a reflectory character (fear, surprise, etc.) are combined with emotions formed after realizing the reason of an emotion arisen (e.g., nostalgia, peacefulness, etc.) and emotions, originated in developed society (e.g., contempt, shame);

- Estimation of emotions is formed as a conscious one, and the border between "emotion" and "feeling" disappeared;

- Objective criteria based on measurement (e.g., relations with neurophysiological reactions) are not applied in emotion scales. The metrological traceability does not take place with regard to the results of quantitative estimations.

\section{Model of measuring emotions as the means for researching the human brain}

The result of measuring expected emotions depends not only on listener's social-cultural features, but also on his/her age. Investigations fulfilled with the help of representative statistical data confirm this fact. However, the causes of the ageing effect on the emotional perception of surrounding world are under discussion because many people are well-known that are distinguished by active emotional life in a very elderly age.

The model developed enables coming to the solution of this effect.

Psychological investigations show that, as applied to music, with age:

- sensitivity to sad and scary music drops [21, 22]; scary music is assessed more positively; sad music is perceived as less sad [23]; negative music causes less emotional tension [24];

- happy music seems a little less happy [23], but overall, the recognition of happiness and peacefulness depends on age only a little [21, 22].

As hypothetical reasons for these changes the authors of [22] call:

- age-related cognitive losses associated with attention and memory;

- age-related sensory distortions associated with worsening vision and hearing;

- neural deterioration in structures involved in the process of emotion processing, and, since distortions in the structures associated with different sensory systems may differ, the recognition of emotions by sight and hearing can also be different;

- change of the top-down processing emotional information (according to the socio-emotional selectivity theory, ageing results in "increased prioritization of goals related to emotional well-being, resulting in increased efforts to allocate cognitive resources towards positive information and away from negative one").

A significant number of publications on this issue indicate its actuality, but there is no experimental evidence of the hypotheses put forward. The mechanism of the age-related change in the recognition of emotions, as well as the mechanism of the emotion formation, remains incomprehensible.

As a first approximation, the deterioration of hearing with age can be characterized by a change in the frequency response of the preselector of the measurement model (Fig.1) as shown in Fig. 3.

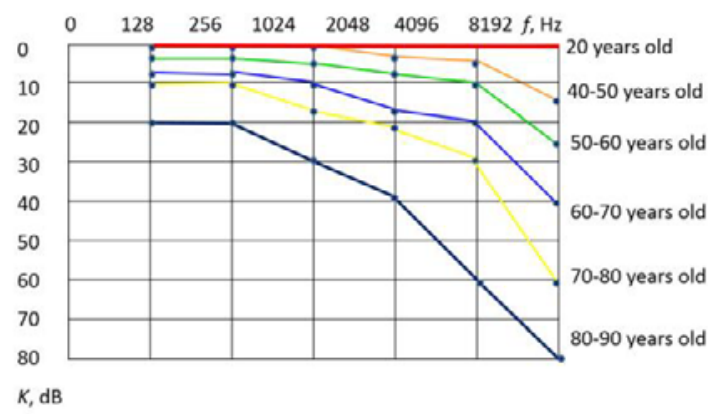

Fig. 3. Decreasing of the hearing sensitivity with age.

Due to the age limitation of the sensitivity of an ear membrane, when a musical fragment, bio-acoustic signals, or speech is sounding, at the output of the non- 
linear converter, as a rule, a smaller number of infrasound intermodulation products that give birth to emotions will be formed [25]. Of course, this effect can be different for various excerpts, the difference being greater if the higher frequency part of the sound range is occupied by the sound spectrum.

The age differences concerning the expected emotions while perceiving a funeral ringing of bells (knell), shown in Fig. 4, are characteristic.

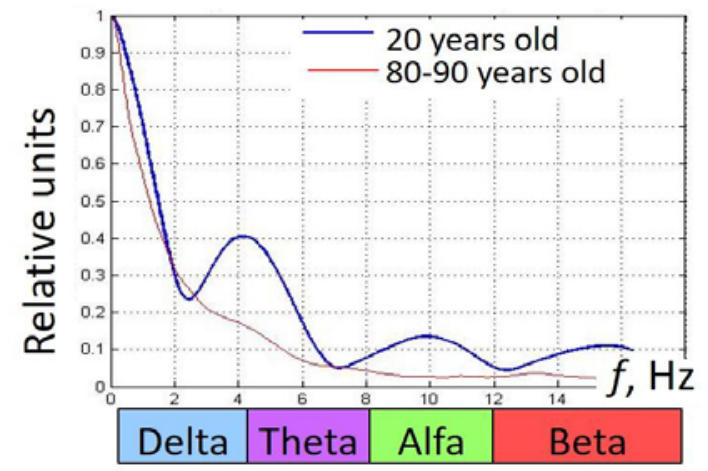

Fig.4. Infrasonic spectrum selected from the music excerpt after non-linear conversion (Funeral ringing of bells (knell).

For a twenty-year-old believer, in this ringing there is an emotion of the fear of death (a rise in the deltarhythm zone), emotion of meeting with something unknown (rise in the theta-rhythm zone), emotion of hope for the best for a dead (rise in the alpha-rhythm zone). However, for the eighty-year-old person all these emotions are smoothed and expressed significantly weaker.

The excerpt presented below in Fig. 5, shows that a twenty-year-old person will perceive much stronger the emotion of fear in a scream of a yellow ground squirrel taken out of a hole. The eighty-year-old person will feel even a tint of a positive coloration in this scream.

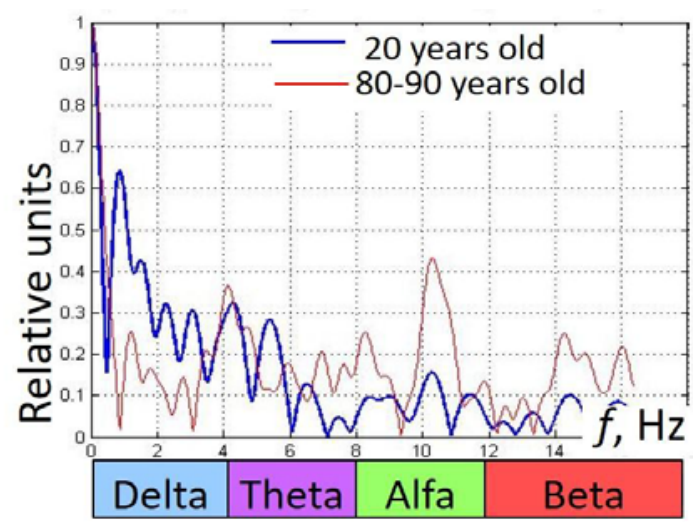

Fig. 5. Infrasonic spectrum selected from a bioacoustics signal excerpt after non-linear conversion (Scream of a yellow ground squirrel taken out of a hole).

Fig.6 is an illustrative example, in which an expected difference in emotional perception of infant's cry is shown. A young potential listener should sharply feel fear (the level of reaction within the delta-rhythm zone is maximum), but an elderly person, practically, should not feel fear. For a young "listener", two different peaks take place in the theta-rhythm zone, the maximum peak is narrower and shifted to the delta-rhythm zone. The last feature will likely stimulate the desire, first of all, to stop infant's cry.

For an elderly person, the difference of similar peaks is not so significant, their maximum being twice less than in the previous case. It seems that the cry will cause alertness and increased attention to an infant, but the actions will be quieter. The difference in the alpharhythm zone makes it possible to suppose that the level of excitation accompanied by corresponding muscle tension should be higher and less smoothed. Such features demonstrate the focus on the specific result.

The proposed interpretation explains the proportion of the infrasound component levels, which stimulate emotiogenic reactions of listeners of different age. At the same time, it is much in line with the most likely emotional reactions and actions.

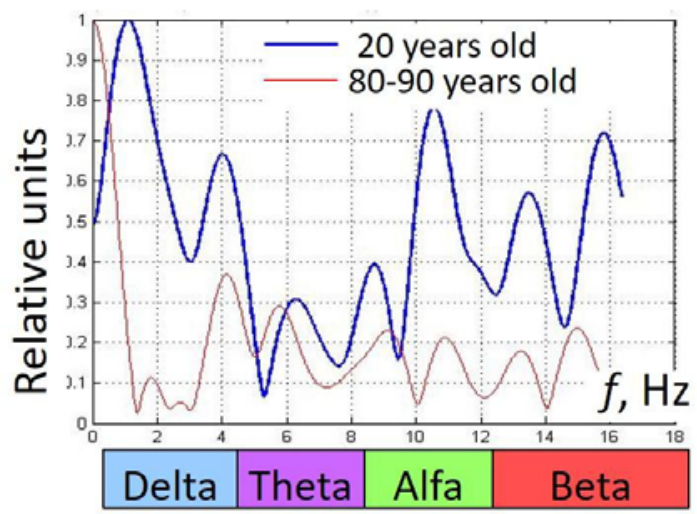

Fig.6. Infrasonic spectrum selected for an infant cry after nonlinear conversion

Similar (qualitatively) age effects are illustrated in Fig. 7 by the infrasound spectrum singled out from the excerpt of "The Little Night Serenade" by V. A. Mozart (a decrease in the energy of the delta- rhythm zone, a slight increase in theta-rhythm zone, a relative equalization of the energy in all the zones studied).

But even in this case, relative changes in the spectrum are noticeably reduced with an increase of the age, causing a drop of the variety of emotional colours being perceived.

The examples given confirm the above observations concerning age influence:

- decrease in the emotion recognition accuracy (decrease in the variety of emotion tints),

- drop of the sensitivity to sounds that are associated with fear,

- and even perception of these sounds as a little more positive.

The origination of the age changes in emotional perception revealed by psychologists can be, to a significant extent, explained by deterioration of hearing. 


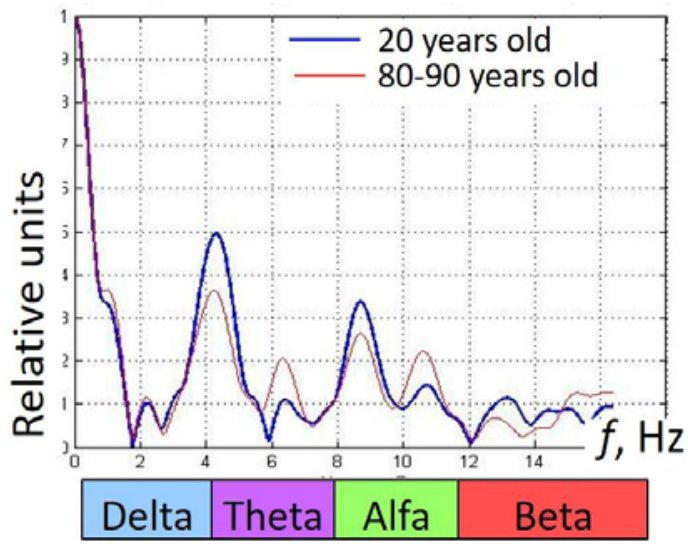

Fig. 7. Infrasonic spectrum selected from a music excerpt after non-linear conversion (V. A. Mozart's "Little Night Serenade").

It can be additionally assumed that the gradual reduction of the flow of infrasonic oscillations in the brain, caused by such a deterioration, leads to a decrease in the sensitivity during the process of forming the neurophysiological reactions that give rise to emotions in a limbic-reticular complex. Accordingly, this assumption makes it possible to explain the drop of the acuity of the reaction while perceiving emotional information entering over other sensory channels.

The example of Beethoven, who fell ill with the titanite and went deaf after writing the Moon Sonata and the Pathetic Sonata, but continued creating masterpieces, provides food for thoughts. Almost completely without hearing, he wrote many musical compositions, including such brilliant creations as the Third (Heroic) and Ninth Symphonies as well as Missa solemnis.

The above facts testify that everything is not so simple with regard to the age changes in the emotional sphere of a person.

If the considered measurement model is analyzed as the model of the "mechanism" of some brain part interacting with other brain parts, it enables offering an explanation for these facts too.

The formation of emotions by means of vision is associated with the recognition of images, their logical comprehension and with the appearance of an emotiogenic reaction only after that. In other words, this process is much more complicated and slow than in case of forming emotiogenic reaction to an acoustic impact. The role of vision in forming the emotional sphere has effectively manifested itself at relatively late stages of evolution.

Unlike the visual impact, music creates a flow of neurophysiological reactions that form the basic emotions in the limbic-reticular complex. First and foremost, it is music that develops the ability to detect differences between various reactions inside this flow and contributes to increasing the sensitivity to these differences in the process of emotion formation.

People who have had a developed emotional sphere since their childhood, often hear "sounds" in their mind.
Caused by music, sequences of neurophysiological reactions from the cerebral cortex, enter the limbicreticular complex, form emotional reactions in it, which returning, are combined with other neurophysiological reactions, forming new emotional images.

That is the way Beethoven created his works.

At that, the neurophysiological reactions entering the limbic-reticular complex, stimulate its work, regardless of where they have originated.

This circumstance makes it possible to explain a sociological phenomenon that has been recently discovered: from a large number of representatives of various specialties, the greatest duration of life distinguishes conductors, artists associated with music and scientists taking great interest in their researches. (The emergence of the latter in this list among the leaders can be associated not only with their rich emotional life, but also with the fact that among them the percentage of active music lovers is high).

Hence, the physiological reasons of music influence on human health become clear. In its turn, their understanding allows formulating arguments that justify the need to apply music at the earliest stages of child upbringing and to develop musical culture of schoolchildren and students, regardless of their future specialization.

Being emotionally saturated throughout the whole life, should result in an increase in life expectancy.

The proved link between acoustic signals with neurophysiological reactions shown in Fig. 1 and Fig. 2 enables proposing a hypothesis about the formation of infant's cry sounding and other vocalizations. According to this hypothesis, the brain emotiogenic reactions (with the frequencies within delta-, theta-, and alpha- brain biorhythms) stimulating basic emotions, affect the nonlinear converter and modulate oscillations with the sound frequencies that also enter this converter.

It follows from this hypothesis that the analysis of cry with the help of the model proposed allows evaluating the brain activity within the infrasound range, which characterize the infant's level of the emotional sphere development. To test the hypothesis experimentally, variation of the sound spectrum converted into the infrasound range was studied with regard to the cry of several infants (girls) 1-2 days and 2 months old.

The results have demonstrated certain trends. During 2 months, the average value of relative power of components with the delta-rhythm frequencies decreased from $59.1 \%$ to $57.5 \%$, with theta-rhythm frequencies increased from $26.4 \%$ to $28.1 \%$, whereas within the alpha-rhythm frequencies this value kept near $14.4 \%$. At the same time, the standard deviation of the value of relative power for the same 2 months increases approximately twice: for delta-rhythms from $3.8 \%$ to $8.9 \%$, for theta-rhythms from $2.9 \%$ to $6.1 \%$, for alpharhythms from $1.6 \%$ to $3.3 \%$ (the higher the frequency, the lower this increase).

The power increase within the theta-rhythm zone reflects the process of emotion understanding.

It is symptomatic that the evolutional development of animal's emotions was associated with consecutive 
growth in neurophysiological activity at higher frequencies.

The standard deviation of the value of biorhythm relative power for a group of infants and its time variation can be considered as parameters characterizing differences related to the level and pace of development of individual infant emotionality features in an infant group under investigation.

Similar parameters obtained for a representative set of vocalizations of an infant and the results of their comparison with the values taken from a reference database can be used as the diagnostic ones. It is important that such an approach enables not only diagnosing diseases, but also detecting deviations that can contribute to development of talents and abilities under some conditions.

\section{Conclusion}

In the coming era of the Internet of Things, measurements of quantities characterizing properties of human should be recognized as one of the most important metrology tasks. In this field, development of measurement models that concern functions of the human organs, especially, the brain, are of special interest. Specialists dealing with various fields of knowledge should take part in these works. Such developments are very complicated, but they open new horizons. In particular, the present paper gives the analysis of some new tasks that can be solved with the help of the model intended for measuring expected emotions caused by acoustic impacts.

A distinctive feature of this model conditioning its efficiency is the fact that it has been developed as a model of "mechanism" of forming neuropsychological reactions causing emotions caused by acoustic impacts. In previous publications, the efficiency of the model was demonstrated for music theory and philology as well as for automatic translation of speech and creative work of musicians.

In the present paper, with the help of the model, the proposed list of earliest (basic) emotions is justified and the zones of biorhythms are determined that activate these emotions.

The model enabled explaining typical changes in emotional music perception with age and long life of representatives of certain professions. The model also made it possible to justify the diagnostic method of infant's emotional development based on sounds of cry and other vocalizations.

The considered approach to the measurement model reflecting the mechanism of forming measurands, has also demonstrated its efficiency for studying some social processes.

The research is supported by the Russian Foundation for Basic Research (RFBR), project "Forming of a measurement model interrelating musical influence and emotions of listeners" (No. 15-04-00565).

\section{References}

1. C.E. Izard, The Psychology of Emotions (Springer US, 1991)

2. G.A. Golitsyn, Information and Creativity (Russkiy Mir, Moscow, 1997)

3. R. Taymanov, Y. Baksheeva, K. Sapozhnikova, A. Chunovkina, JPCS, $772 \quad$ (2016). http://iopscience.iop.org/article/10.1088/17426596/772/1/012036

4. Y. Baksheeva, O. Bazanova, S. Kostromina, E. Nikolenko, K. Sapozhnikova, R. Taymanov, Proc. the Ninth Triennial Conference of the European Society for the Cognitive Sciences of Music (Manchester, UK, 2015)

5. Yu. Baksheeva, K. Sapozhnikova, R. Taymanov, Advances in Mathematical and Computational Tools in Metrology and Testing X. Series on Advances in Mathematics for Applied Sciences, 86, 90-97 (Singapore, World Scientific, 2015a)

6. K. Sapozhnikova, R. Taymanov, Proc. XVII IMEKO World Congress, 2049-2053 (Dubrovnik, Croatia, 2003)

7. K. Sapozhnikova, R. Taymanov, Measurement Science Review, 10, No 3, 78-88 (2010)

8. R. Taymanov, K. Sapozhnikova, EPJ Web of Conferences, $77 \quad$ (2014). http://www.epjconferences.org/articles/epjconf/pdf/2014/14/epjcon f_icm2014_00016.pdf

9. R. Taymanov, K. Sapozhnikova, KEM, 613, 482491 (2014)

10. R. Taymanov, Y. Baksheeva, K. Sapozhnikova, Proc. 12th International Symposium on Measurement Technology and Intelligent Instruments (ISMTII 2015) (Taipei, Taiwan, 2015)

11. P. Ekman, Emotions Revealed: Recognizing Faces and Feelings to Improve Communication and Emotional Life [Psikhologiya emotsiy. Ya znayu, chto ty chuvstvuesh] (St. Petersburg, Piter, 2010)

12. R. Plutchik, Annul. N.-Y. Acad.Science, 134, No 2, 776-781 (1966)

13. L.V. Kulikov, Psychology of Mood [Psihologiya nastroeniya] (St. Petersburg, 1997)

14. E.P. Il'in, Emotions and Feelings [Emotsii $i$ chuvstva] (St. Petersburg, Piter, 2013)

15. K. Sapozhnikova, R. Taymanov, Proc. of the 10th International Symposium on Measurement Technology and Intelligent Instruments (ISMTII2011) (Daejeon, Korea, 2011)

16. M. Zentner, D. Grandjean, K.R. Scherer, Emotion, 8, No 4, 494-521 (2008)

17. E. Coutinho, K.R. Scherer, Proc. of the 12th International Conference of Music Perception and Cognition (ICMPC12) (Thessaloniki, Greece, 2012)

18. Basic Emotions.

http://changingminds.org/explanations/emotions/bas ic\%20emotions.htm 
19. T. Eerola, J.K. Vuoskoski, Music Perception, 30, No 3, 307-340 (2013)

20. J. Russell, A. Weiss, G. Mendelsohn, J. Personality and Soc. Psychol., 57, No 3, 493-502 (1989)

21. C.F. Lima, S.L. Castro, Cognition and Emotion, 25 (4), 585-598 (2011)

22. S.L. Castro, C.F. Lima, Music Perception, 32 (2), 125-142 (2014)
23. M.T. Pearce, A.R. Halpern, Psychol. of Aesthetics, Creativity, and the Arts, 9, No 3, 248-253 (2015)

24. S. Vieillard, J. Harm, E. Bigand, Frontiers in Aging Neuroscience, 7 (FEB), art. no. 11, 1-14 (2015)

25. K. Sapozhnikova, I. Baksheeva, R. Taymanov, A. Tanonov, Proc. Symposium on music research "Music, ageing, technology" (Joensuu, Finland, 2016) 